Casos Clínicos

Arch. Esp. Urol. 2010; 63 (6): 477-479

\section{HISTIOCITOMA FIBROSO MALIGNO RETROPERITONEAL. A PROPÓSITO DE UN CASO}

Pablo Eguiluz Lumbreras, Alberto Palacios Hernández, Oscar Heredero Zorzo, Javier García García, Florencio Cañada de Arriba, Federico Pérez Herrero y Ramón Gómez Zancajo.

Servicio de Urología. Hospital Clínico Universitario de Salamanca. Salamanca. España.

Resumen.- OBJETIVO: Presentar un caso de una tumoración retroperitoneal de gran tamaño con diagnóstico patológico de Histiocitoma fibroso maligno y revisar aspectos diagnósticos y terapéuticos de la literatura actual.

MÉTODO: Presentamos el caso de un hombre de 75 años de edad que durante un ingreso en el servicio de digestivo por clínica de astenia de meses de evolución y problemas gastrointestinales se realiza CT abdomino-pélvico, identificando gran masa de $20 \times 22 \times 12 \mathrm{~cm}$ que parece depender del riñón izquierdo junto a aneurisma de aorta abdominal de $8 \mathrm{~cm}$ de diámetro.

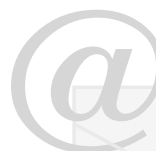

\section{CORRESPONDENCIA}

Pablo Eguíluz Lumbreras

Henry Collet, 14-16-Portal: 5 2C. 37007 Salamanca (España).

paeglu@hotmail.com

Aceptado para publicar: 17 de noviembre 2009.
RESULTADOS: Se somete al paciente a intervención quirúrgica donde se realiza Nefrectomía radical izquierda más resección radical de masa retroperitoneal, que histológicamente corresponde a un Histiocitoma fibroso maligno, tipo estoriforme-pleomórfico con focos de degeneración hialina (Estadio pT2b).

CONCLUSIONES: Los sarcomas son neoplasias infrecuentes. Pueden adoptar una gran variedad de patrones morfológicos y distintos grados de diferenciación. El tratamiento quirúrgico continúa siendo la única terapia con posibilidades curativas. El tratamiento adyuvante radioterápico y/o quimioterápico es cuestionado.

Palabras clave: Histiocitoma fibroso maligno. Retroperitoneo. Cirugía.

Summary.- OBJECTIVE: We present the case of a big retroperitoneal tumor that received the pathologic diagnosis of malignant fibrous histiocytoma. We also review the diagnostic and therapeutic features of this disease in the current literature.

METHODS: We present the case of a 75-year-old male who was admitted to the Gastrointestinal Disease Department with asthenia of several months of evolution and gastrointestinal problems. Abdominopelvic CT scan revealed a big mass of $20 \times 22 \times 12 \mathrm{~cm}$, which seems to depend from the left kidney, together with an $8 \mathrm{~cm}$ diameter abdominal aortic aneurysm.

RESULTS: The patient underwent surgery and left radical nephrectomy together with radical resection of the retroperitoneal mass were performed. Pathology reportes malignant fibrous histiocytoma of the storiform-pleomorphic type, with hyaline degeneration foci (stadium PT2B).

CONCLUSIONS: Sarcomas are rare neoplasias. They can adopt several different morphologic patterns, as well as many differentiation degrees. The surgical treatment is still the only therapy with healing possibilities. Adjuvant treatments through radiotherapy and/or chemotherapy are brought into question.

Keywords: Malignant fibrous histiocytoma. Retroperitoneo. Surgery.

\section{INTRODUCCIÓN}

Las masas retroperitoneales son neoformaciones benignas o malignas originadas en las distintas estructuras que conforman este espacio, sin incluir los órganos situados en él. El $85 \%$ de los casos son malignos, de los que $35 \%$ son una tumoración rara. 
El histiocitoma fibroso maligno forma parte de los sarcomas de partes blandas, pueden surgir en cualquiera de los tejidos mesodérmicos de las extremidades $(50 \%)$, del tronco y el retroperitoneo $(40 \%)$, o de la cabeza y el cuello $(10 \%)$. Es un tumor muy infrecuente en la infancia y más frecuente en hombres que en mujeres. Su origen aún está en discusión aunque parece derivar de células mesenquimales pluripotentes.

\section{CASO CLÍNICO}

Paciente varón de 75 años de edad, con HTA y Dislipemia como únicos antecedentes médicos de interés, en tratamiento con Doxazosina y Trimetazidina. Como antecedentes familiares de interés cabe destacar un cáncer de vejiga en su padre y un cáncer de pulmón en su madre que provocó su fallecimiento. El paciente acudió a su médico de atención primaria por clínica de astenia y pérdida de peso de 4 meses de evolución. Su médico de atención primaria solicitó una analítica sanguínea en la que observó unos depósitos de ferritina disminuidos tras los cuales pautó tratamiento con hierro. El paciente acude a urgencias por problemas gastrointestinales donde le realizan analítica de control con unos datos analíticos normales salvo una hemoglobina de $10.5 \mathrm{~g} / \mathrm{dl}$, hematocrito de $33,5 \%$, y radiografía de tórax en la que se observa una elongación de la aorta. Se ingresa al paciente a cargo de servicio de digestivo y se realiza un estudio de marcadores tumorales sanguíneos CEA, Ca 19.9 y alfafetoproteína, siendo todos ellos normales y un CT Abdomino-pélvico con contraste intravenoso para completar el estudio.
En el CT se descubre una masa de $20 \times 22 \times 12 \mathrm{~cm}$ que parece depender del riñón izquierdo y un aneurisma abdominal de $8 \mathrm{~cm}$ de diámetro (Figura 1). Tras este hallazgo radiográfico se remite al paciente a consulta de Urología y tras realizar sesión conjunta con el servicio de Cirugía Vascular se decide intervención quirúrgica un mes tras el diagnóstico en la que se realiza una nefrectomía radical izquierda con la resección radical de la masa retroperitoneal de $20 \times 17 \times 13 \mathrm{~cm}$ de diámetro y un Bypass aortoiliaco derecho.

El estudio histológico describe una masa tumoral que microscópicamente corresponde a un tumor mesenquimal maligno con rasgos histológicos e inmunohistoquímicos indicadores de: Histiocitoma fibroso maligno, tipo estoriforme-pleomórfico con focos de degeneración hialina (Estadio pT2b) (Figura 2).

En el postoperatorio inmediato el paciente sufrió un síndrome coronario agudo que fue tratado médicamente por el servicio de cardiología detectado por una elevación ligera de las enzimas cardiacas. Durante el quinto día de ingreso hospitalario sufrió un episodio de hipertermia de $40^{\circ} \mathrm{C}$, acompañado de sudoración profusa y abundante expectoración. A la auscultación pulmonar se observa disminución de murmullo vesicular en base pulmonar izquierda por lo que se solicita radiografía de tórax, que descarta un posible proceso neumónico. Durante los días posteriores el paciente permaneció apirético y tanto hemodinámica como clínicamente bien, siendo dado de alta médica a los 10 días tras la intervención con un ligero deterioro de la función renal $(\mathrm{Cr}$ : $1.36 \mathrm{ngr} / \mathrm{ml}$ ) y con el resto de parámetros analíticos,

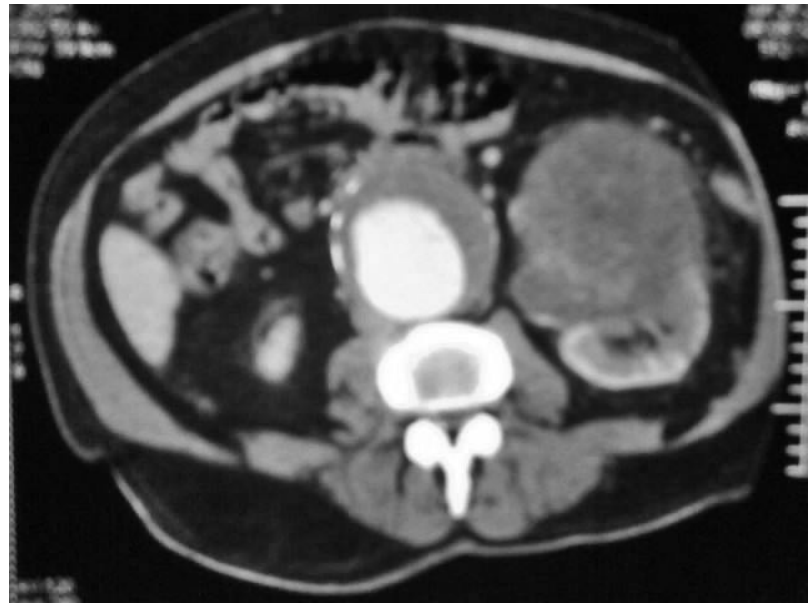

FIGURA 1. CT Abdomino-Pélvico: Evidencia de gran masa retroperitoneal izquierda de $20 \times 17 \times 13 \mathrm{~cm}$ de diámetro que desplaza estructuras vasculares y aneurisma aorta abdominal.

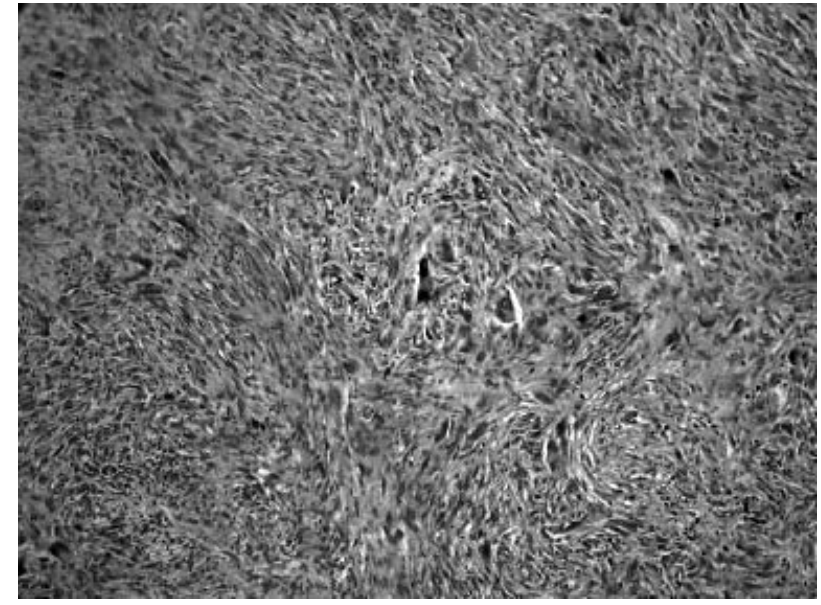

FIGURA 2. Anatomía patológica: Tumor mesenquimal maligno con rasgos histológicos e inmunohistoquímicos indicativos de histiocitoma fibroso maligno de tipo estoriforme-pleomórfico predominante, con focos de degeneración mixoide-hialina, así como de áreas de necrosis y hemorragia. 


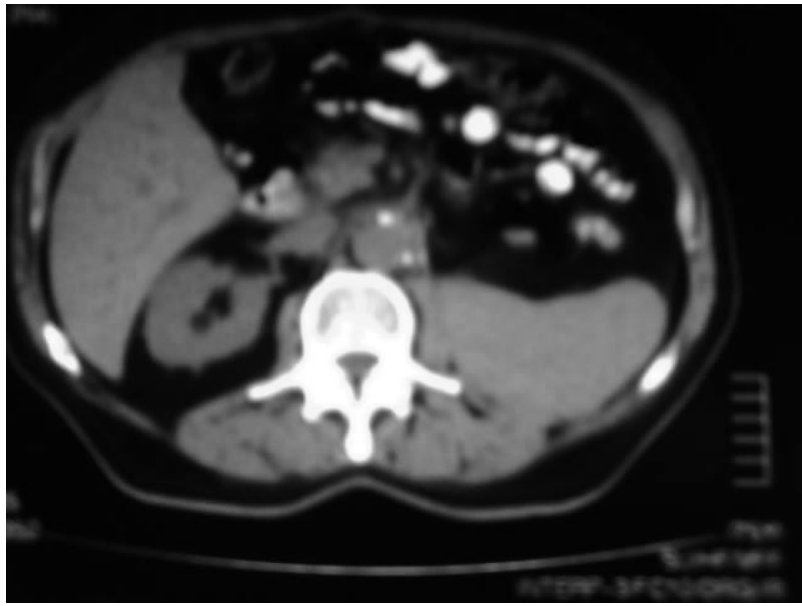

FIGURA 3. CT Toraco-Abdomino-Pélvico postlQ: Cambios postquirúrgicos tras nefrectomía izquierda. No se identifican adenopatías abdomino-pélvicas.

(hemograma, bioquímica y pruebas de coagulación) dentro de los rangos de la normalidad.

Tras extirpación radical de la masa se envía al paciente al servicio de Oncología donde desestiman tratamiento adyuvante reenviándolo a Oncología Radioterápica donde se decide abstinencia terapéutica.

A los 3 meses de la intervención se realiza revisión en consulta externa de Urología, donde el paciente nos comunica encontrarse clínicamente bien. Se efectúa control analítico encontrándose todos los valores dentro de la normalidad y CT Toraco-abdomino-pélvico donde se observan adenopatías mediastínicas múltiples de 1 $\mathrm{cm}$ aprox., aneurisma de aorta torácica de $6 \mathrm{~cm}$ de diámetro, lesiones hipodensas en hígado que impresionan de quistes simples, riñón derecho normal y cambios postquirúrgicos en zona de nefrectomía izquierda y en aorta infrarrenal con Bypass femoral izquierdo permeable (Figura 3).

\section{CONCLUSIÓN}

Los sarcomas son neoplasias infrecuentes, la mayor incidencia se sitúa entre la quinta y la séptima décadas de la vida, siendo inusual su presentación antes de los 40 años (1). Pueden adoptar una gran variedad de patrones morfológicos y distintos grados de diferenciación, según la célula de la que proceden o la estirpe hacia la que se diferencien. Generalmente afectan a partes blandas profundas, en las extremidades y el retroperitoneo, aunque pueden aparecer en cualquier localización. Estos tumores presentan agresividad fundamentalmente local, con marcada tendencia a la recidiva y es infrecuente la aparición de metástasis ganglionares linfáticas.
El Histiocitoma Fibroso Maligno es uno de los pocos sarcomas que se puede presentar como un fumor superficial, y puede mostrar un patrón mixoide (con abundante matriz de mucopolisacáridos ácidos), estoriforme-pleomórfico, de células gigantes, o de predominio inflamatorio (con células xánticas y neutrófilos), siendo el patrón estoriforme el más frecuente (2). Debido a que la presencia de un patrón predominantemente mixoide se asocia a un mejor pronóstico, se recomienda distinguir en el diagnóstico si se trata de un HFM de variante mixoide o no mixoide (3). Del mismo modo, también implican menor riesgo de metástasis a distancia un tamaño tumoral pequeño, la localización superficial y la presencia de prominente infiltrado inflamatorio (4), siendo la existencia de márgenes quirúrgicos menores de 2 $\mathrm{cm}$ el principal factor a considerar para determinar el riesgo de recidiva local, metástasis a distancia o tiempo de supervivencia (5).

El tratamiento quirúrgico continúa siendo la única terapia con posibilidades curativas, aunque para ello sea necesaria la resección completa con unos márgenes de seguridad de $2 \mathrm{~cm}$.

Recurrencia: 50-82\% dependiendo del grado, tamaño y estadio del tumor. La localización más frecuente de afectación a distancia es por orden decreciente: Pulmón, hígado, hueso y M.O.

El tratamiento adyuvante radioterápico y/o quimioterápico (adriamicina sola o en asociación con DIC y vincristina), es cuestionable, no parece aumentar la supervivencia pero si aumenta el periodo libre de enfermedad.

\section{BIBLIOGRAFÍA y LECTURAS RECOMENDADAS ( ${ }^{*}$ lectura de interés $y^{* *}$ lectura fundamental)}

**1. Giménez Bachs JM, Salinas Sánchez A, Lorenzo Romero J, Donate Moreno MJ, Hernández Millan IR, Segura Martín M et al. Fibrohistocitoma maligno retroperitoneal de gran tamaño: aportación de un caso. Arch Esp Urol. 2004;57(2): 172-4

2. Weiss SW, Goldblum JR. Malignant fibrohistiocytic tumors. En: Enzinger and Weiss's Soft tissue tumors. 4th ed. Mosby, St Louis. 2001. p 535-69.

3. Weiss SW, Enzinger FM. Myxoid variant of malignant fibrous histiocytoma. Cancer 1977; 39: 1672-85.

4. Weiss SW, Enzinger FM. Malignant fibrous histiocytoma: an analysis of 200 cases. Cancer 1978; 41: 225066.

5. Yamaguchi S, Nagasawa H, Suzuki T, et al. Sarcomas of the oral and maxillofacial region: a review of 32 cases in 25 years. Clin Oral Investig 2004; 8: 52-5. 